\title{
An Anatomical Perspective of Ulnar Collateral Nerve with Reference to Nerve and Muscle Transfer Surgery
}

\author{
P. J. Jiji., T. Joy, B.V. Murlimanju, M. M. Pai, L. V. Prabhu, R. Rai \\ Department of Anatomy, Kasturba Medical College, Mangalore, Manipal Academy of Higher Education, Manipal, \\ Karnataka, India
}

\author{
CORRESPONDING AUTHOR: \\ Teresa Joy \\ Department of Anatomy \\ Kasturba Medical College \\ Mangalore, India \\ Manipal Academy of Higher Education \\ 575004 Karnataka, India \\ E-mail: teresa.joy@manipal.edu
}

DOI:

10.32098/mltj.03.2020.23

LEVEL OF EVIDENCE: 4

\begin{abstract}
SUMMARY
Background. The purpose was to analyze the prevalence, origin, course and termination of the ulnar collateral nerve in anatomical samples from Indian population.

Methods. The present study included 68 adult human cadaveric embalmed upper limbs. The sex of these upper limbs were not known. The initial segment of radial nerve was meticulously dissected in the specimens to observe the ulnar collateral nerve. The digital Vernier caliper was used for the measurements which were performed in this study. The data was given in millimeter and expressed as mean \pm SD.

Results. The ulnar collateral nerve was observed in $57(83.8 \%)$ of our specimens. It originated in the axilla in 35 cases $(61.4 \%)$, at the brachio-axillary angle in $13(22.8 \%)$ and in the arm in 9 cases $(15.8 \%)$ respectively. The termination of ulnar collateral nerve by piercing deep into the triceps muscle happened $101.9 \pm 4 \mathrm{~mm}$ above the medial epicondyle of humerus. The origins of muscular branch to long head of triceps muscle and ulnar collateral nerve were located $44.7 \pm 3.7 \mathrm{~mm}$ and $66.7 \pm 4.4 \mathrm{~mm}$ away from the bifurcation of posterior cord respectively.

Conclusions. The present study suggests higher frequency of ulnar collateral nerve in sample Indian population. Ulnar collateral nerve can be used to re-innervate the muscles in case of brachial plexus injuries. Our study provides information about the detailed morphology and topography of the ulnar collateral nerve. The detailed knowledge can help the orthopedic surgeons during the procedures like muscle transfer, nerve transfer and nerve grafting.
\end{abstract}

\section{KEY WORDS}

Elbow surgery; radial nerve; triceps brachii; ulnar collateral nerve.

\section{BACKGROUND}

Variant innervations of muscles of upper extremity are comparatively rare, but if present will have clinical, diagnostic and surgical implications (1). Triceps brachii is conventionally believed to be dependent on the radial nerve (RN) for its motor innervation, but few studies (1-6) has confirmed the ulnar innervation for the distal part of its medial head. But there are older descriptions that says there were no branches given by ulnar nerve (UN) in the arm and thus entire nerve supply of triceps is by RN (7). However there are cases reported in which axillary nerve supplied the triceps brachii (8). Naidu et al. (9) revealed the independent function of lateral and long heads of triceps brachii in their electromyographic studies. They restored the flexion of elbow by triceps to biceps tendon transfer procedure. The distal part of medial head of triceps is often affected in subluxation injuries. Posterior approach is commonly performed for the elbow surgery and this requires triceps splitting and triceps reflection (10). In this context, it is important to have the knowledge about anatomical variations in the innervation of triceps brachii. This will prevent inadvertent iatrogenic injury and also provides new modalities for the free functional nerve and muscle transfers for the triceps brachii (11).

Ulnar collateral nerve (UCN) runs along with the UN, but it is a branch of RN (12-14). UCN disappears in the back 
of arm by piercing the medial head of triceps brachii ${ }^{15}$ and innervates it (16). It was described earlier that the UCN is a sensory nerve, though it pierces medial head of triceps brachii (17). Later, the immunohistochemical studies showed that the UCN is not purely a sensory or motor nerve, it is a mixed nerve (16). UCN also supplies the proximal part of the posterior part of capsular ligament of elbow (18). In brachial plexus injuries, de-innervated muscles can be re-innervated by using the UCN (2). The muscle unit along with its innervation may be transferred to reestablish the flexion of elbow (19). Nerve transfer procedures performed for the brachial plexus reconstruction and peripheral nerve injuries have given better outcomes than the nerve grafting (20). Literature search revealed that there are only a very few studies available about the UCN. This nerve is not highlighted in the anatomical discussions and the morphological data about this nerve are scarce from the Indian population. This was the stimulus to perform this present study. Our goal was to analyze the prevalence, origin, course and termination of the UCN in cadaveric samples.

\section{MATERIALS AND METHODS}

The present study included 68 adult human cadaveric embalmed upper limbs with intact axillary region. Among them 43 were right sided and 25 left sided. The source of cadavers is from body donation and unclaimed bodies from the government hospital. The present anatomical investigation has the approval of our institutional ethics committee. We also state that this study is in accordance with the international ethical standards, as per the opinion of Padulo et al. (21). The specimens which exhibited surgical scars, external pathological changes and congenital anomalies were excluded from the present study. The upper limbs were positioned with approximately $90^{\circ}$ of abduction. The origin, course and termination of UCN was carefully dissected and observed in all the cases. The dissection was performed as per the standard steps given in the manuals of human anatomy. The nerve supply of the medial head of triceps muscle was observed in all the upper extremities. The topographical location of origin of UCN was classified into 3 categories, in the axilla, at the brachio-axillary angle and in the arm as per Hollinshead (12). The distance of origin of UCN was measured from the inferior margin of latissimus dorsi tendon close to its insertion. The topographical location of UCN, where it enters the triceps at its medial head was measured from the tip of the medial epicondyle, with a digital Vernier calliper. The distances of origins of muscular branch to long head and UCN, from the termination of posterior cord, were also measured. The data was given in millimeter and expressed as mean \pm SD.

\section{RESULTS}

The present study observed the UCN in 57 (83.8\%) cases. Among them 36 (63.2\%) were observed in right side upper limbs and $21(36.8 \%)$ over the left side. The UCN was absent in 11 upper limbs (16.2\%). It was observed that UCN was arising as the first robust branch to the medial head from the RN and coursing with the UN in the anterior brachium. UCN was innervating the medial head of triceps in each upper extremity, whenever present. The origin of UCN was ranging from $40 \mathrm{~mm}$ above and $40 \mathrm{~mm}$ below the inferior margin of latissimus tendon. It was macroscopically observed that the UCN presented a separate fascial sheath as it coursed close to the UN. We did not observe UN supply $(0 \%)$ to triceps brachii muscle in this study. Frequency of topographical distribution of the origin of UCN is represented in figure 1. The UCN was originating in the axilla (figures 2,3) in 35 cases $(61.4 \%$ ), at the brachio-axillary angle (figure 4$)$ in $13(22.8 \%)$ and in the arm (figure 5) in 9 cases $(15.8 \%)$ respectively.

Observation of initial segment of $\mathrm{RN}$ revealed that its first muscular branch was to the long head of triceps brachii (figure 2) and was observed in 65 (95.6\%) of upper limbs. In $18(26.5 \%)$ cases, at the entry of spiral groove, $\mathrm{RN}$ gave a couple of branches, one each to the lateral and medial head of triceps (figure 5). In 22 (32.4\%) upper limb specimens, proximal part of medial head of triceps received additional branches either from RN or UCN (figure 3).

It was observed that the topography of termination of UCN by piercing deep to the medial head of triceps brachii was extremely variable. This was ranging between $50.5 \mathrm{~mm}$ to $180.5 \mathrm{~mm}$, superiorly to the tip of the medial epicondyle, and the mean distance was $101.9 \pm 4 \mathrm{~mm}$. The origins of muscular branch to long head and UCN were located 44.7 $\pm 3.7 \mathrm{~mm}$ and $66.7 \pm 4.4 \mathrm{~mm}$ from the termination of posterior cord respectively.

The anatomical scheme of the complex variant anatomy of UCN, which was observed in this present study is represented in Fig. 6. The frequency of UCN, which was observed in the present study was compared with the data available in the literature (table I).

\section{DISCUSSION}

$\mathrm{RN}$ is the thickest branch which arises from the posterior cord, which usually provides three branches in the axilla -two muscular and one cutaneous, the cutaneous branch being the posterior cutaneous nerve of arm. The long head of triceps receives the first muscular twig and the second twig goes to the medial head, which is the UCN. RN provides more muscular twigs as it enters the spiral groove -one twig supplies the lateral head and another given to the medial 


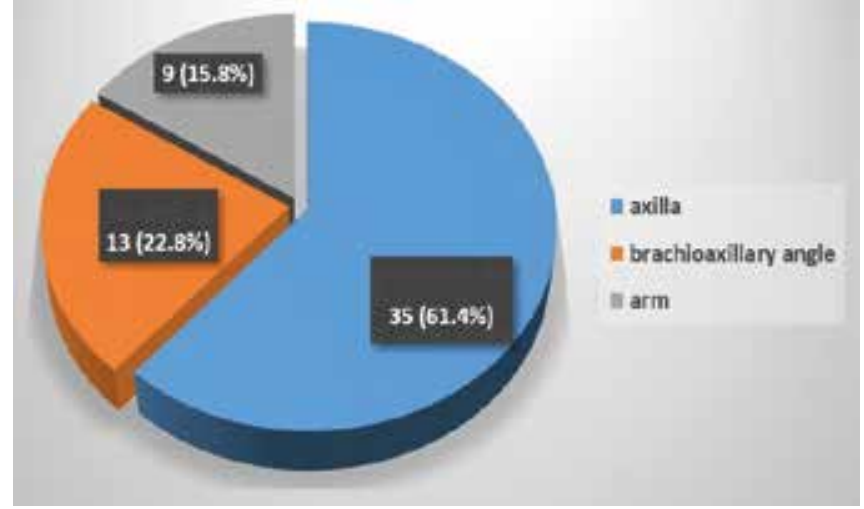

Figure 1. Frequency of topographical distribution of the origin of UCN $(n=57)$.

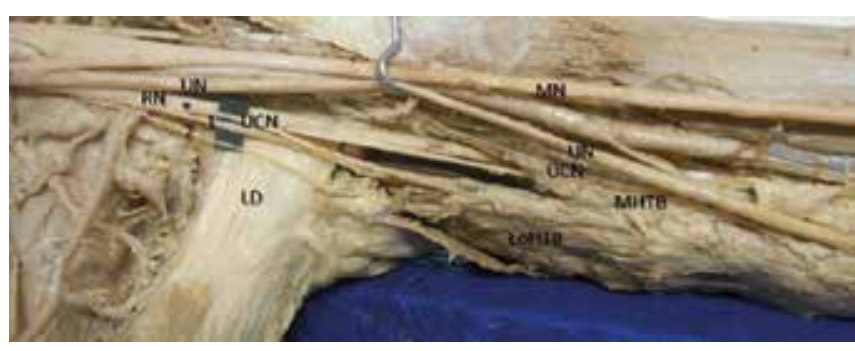

Figure 2. Cadaveric upper limb showing the origin of UCN in the axilla, $61.4 \%$ cases.

(*- origin of UCN; RN- radial nerve; UN- ulnar nerve; UCNulnar collateral nerve; $\mathrm{MN}$ - median nerve; LD- latissimus dorsi; LoHTB- long head of triceps brachii; MHTB- medial head of triceps brachii; 1- branch of radial nerve for LoHTB; 2-posterior cutaneous nerve of the arm).

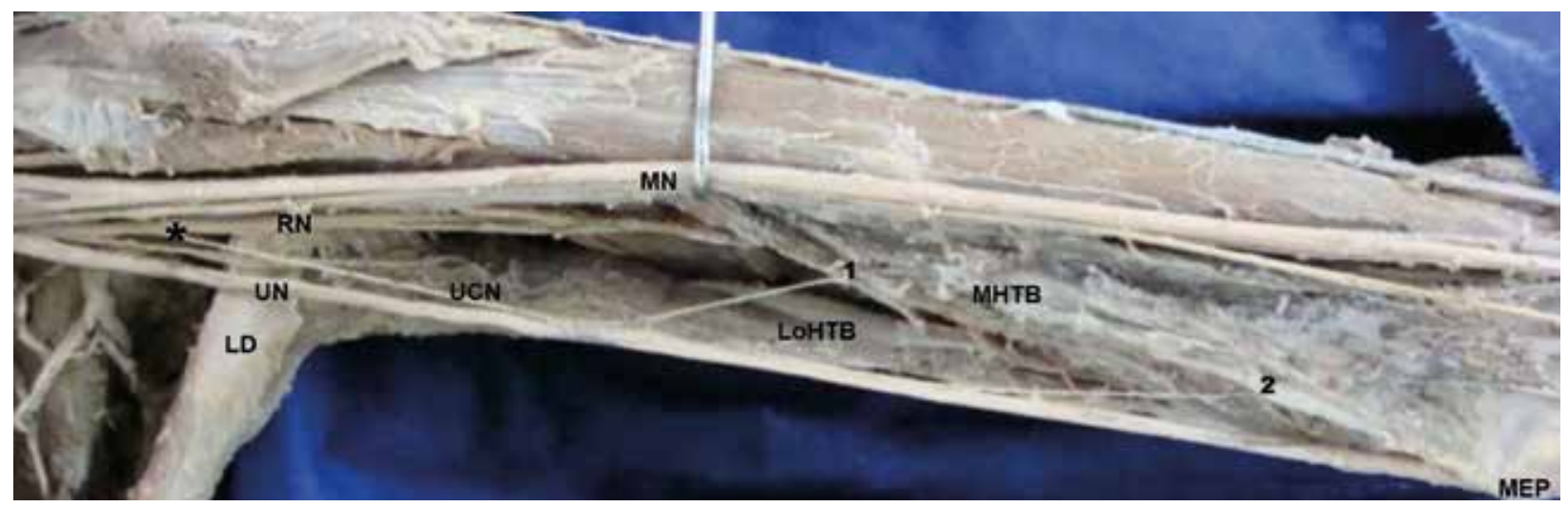

Figure 3. Cadaveric upper limb showing the multiple branches of UCN innervating the medial head of triceps, $32.4 \%$ cases. (*- origin of UCN; RN- radial nerve; UN- ulnar nerve; UCN- ulnar collateral nerve; MN- median nerve; LD- latissimus dorsi; LoHTB- long head of triceps brachii; MHTB- medial head of triceps brachii; MEP- medial epicondyle; 1,2- branches from UCN innervating the MHTB).

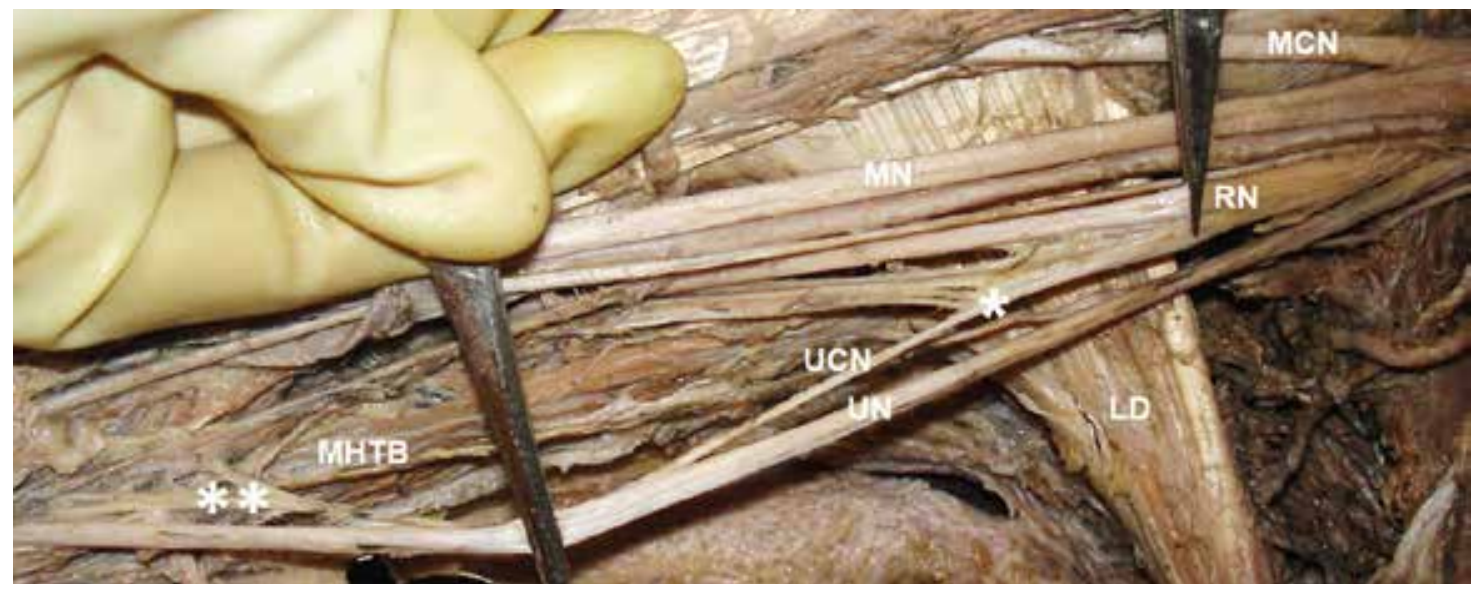

Figure 4. Cadaveric upper limb showing the origin of UCN in the brachio-axillary angle, $22.8 \%$ cases.

(*- origin of UCN; RN- radial nerve; UN- ulnar nerve; UCN- ulnar collateral nerve; MN- median nerve; MCN- musculocutaneous nerve; LD- latissimus dorsi; MHTB- medial head of triceps brachii; ${ }^{\star *}$ - UCN piercing MHTB and innervating it). 


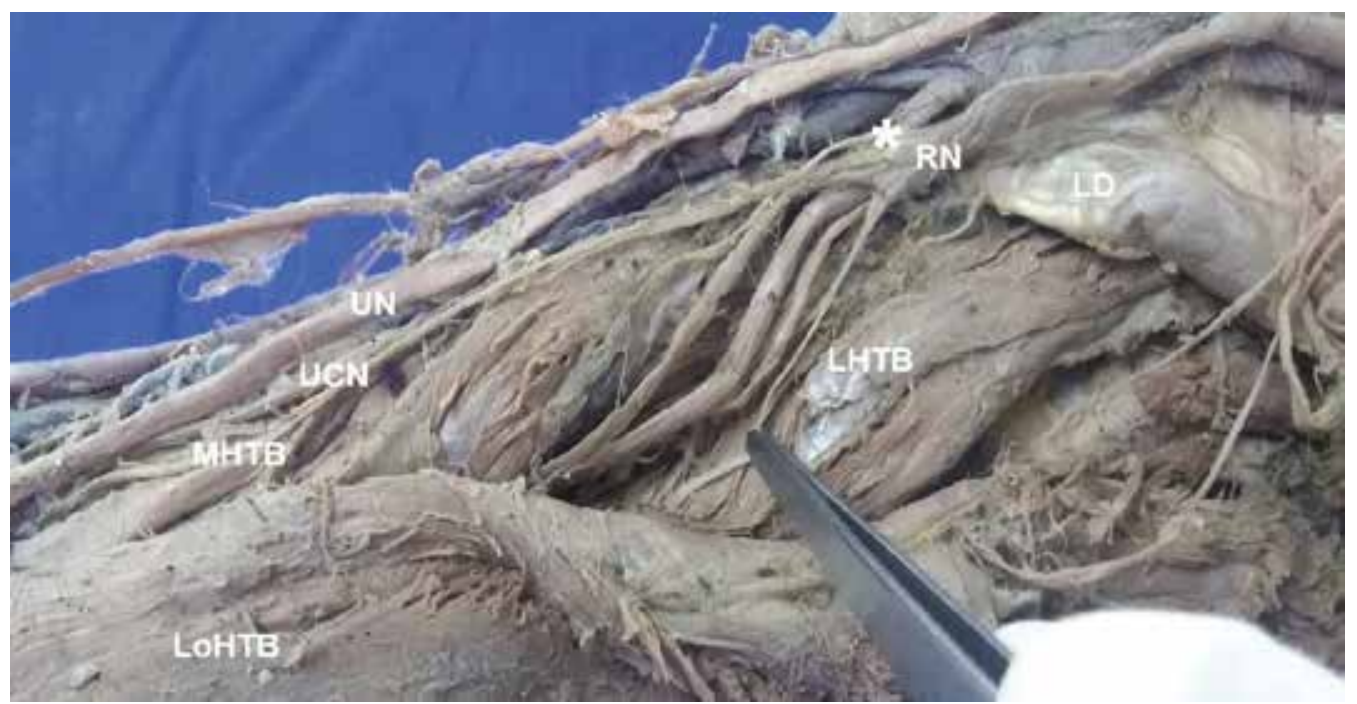

Figure 5. Cadaveric upper limb showing the origin of UCN in the arm, $15.8 \%$ cases. (*- origin of UCN; RN- radial nerve; UN- ulnar nerve; UCN- ulnar collateral nerve; LD- latissimus dorsi; MHTB- medial head of triceps brachii; LHTB- lateral head of triceps brachii; LoHTB- long head of triceps brachii).

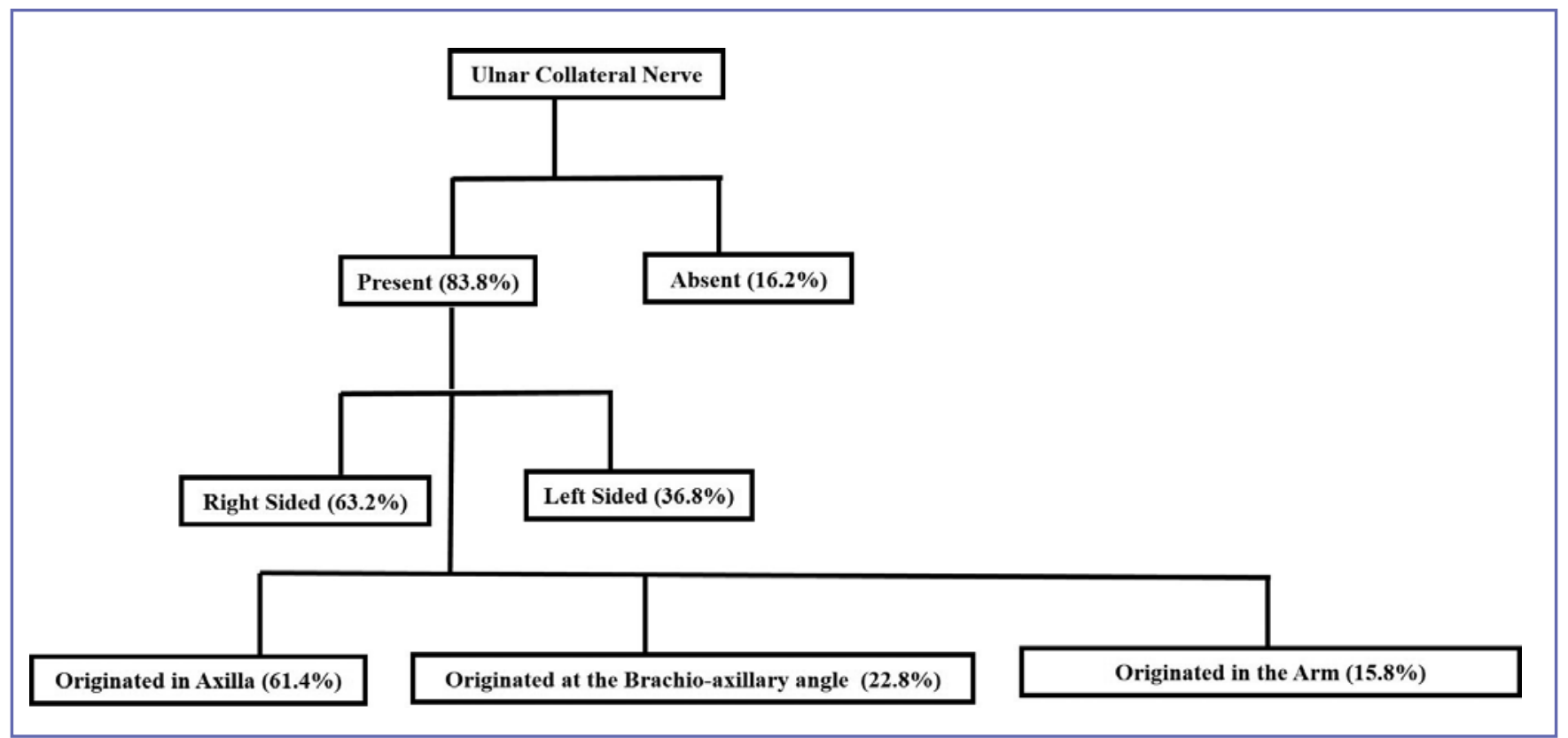

Figure 6. The anatomical scheme of the complex variant anatomy of UCN, which was observed in this study.

head which is considered as major innervation to the medial head (12). According to Linell (17) nerve to long head arises about $7.1 \mathrm{~cm}$ below the tip of acromion, the UCN about 9.5 $\mathrm{cm}$, the nerve to lateral head about $10.1 \mathrm{~cm}$ and the major nerve to medial head about $11.2 \mathrm{~cm}$ below the tip of acromi- on process. In this present study, the origin and termination of UCN was measured from the terminal part of posterior cord and medial epicondyle respectively. Sunderland (22) agreed with the opinion of Linell (17) about the presence of these four branches of $\mathrm{RN}$ but observed five to ten twigs. 
In $32.4 \%$ of cases of present study, it was observed that $\mathrm{RN}$ was giving multiple additional twigs to medial head.

According to Johnson and Ellis (13) and Sinnatamby (14), $\mathrm{RN}$ gives its major twig to the medial head close to its entry into the radial groove. However many twigs can be given to the medial head at the axilla and proximal part of arm. The first RN branch to medial head is the UCN and this is given usually in the axilla or as it enters the arm (12). Our present study agrees with the description of Hollinshead (12) as it was observed that the first muscular twig to medial head was UCN which was found in $83.8 \%$ cases. This frequency of UCN was compared with the global data available in the literature. This rate of $83.8 \%$ from Indian population is slightly higher than that of Turkish (4) (60\%) and Korean (5) $(75 \%)$ population. But lesser than Spanish ${ }^{16}$ population of $100 \%$ prevalence (table I).

It was described that the motor supply to the medial head of triceps is complex and controversia $(14,11)$. The UN supply to the distal third of medial head was also advocated by few people in recent years (1-6). It was described that these UN twigs were confined to the distal third of the triceps and were hardly discernible from the fascia to be established macroscopically. The concept of UN supply to the triceps was questioned by Pascual-Font et al. (16). from their study on foetal specimens. Their study augmented with immunohistochemistry, reported that triceps was supplied by $\mathrm{RN}$ alone. They had further commented that while planning muscle or nerve transpositions by counting on the probable UN supply to medial head, the actual origin should be checked to minimize the failure of the procedure. Pascual-Font et al. (16). had further commented that prior confirmation of the real origin of innervation of the medial head, whether it is ulnar or radial is essential for the procedures like muscle and nerve transposition. This helps to prevent the failure of the transposition when performed. In their study, the UCN was present in all of their cases $(100 \%)$ and it was a branch of $\mathrm{RN}$ but coursing along with the UN without intermingling of fibres but sharing a common neural sheath. Our study agrees with this opinion as we did not observe the UN supply of the medial head in any of our specimens. It may be also possible that those could be extremely thin branches and were not discernible from fascial sheath in dissections. It is interesting to note that medial head of triceps inserts separately in a deeper plane at the olecranon process along with the superficial insertion produced jointly by long and lateral heads (23). If there is ulnar innervation of triceps muscle, the clinical implication will be to include the medial head besides the long head of triceps, while performing triceps-to-biceps tendon transfer surgery (6).

The clinical use of RN branch to long head of triceps for nerve reconstruction in brachial plexus injures was initial- ly performed by Leechavengvongs et al. (24). Develi (4) reported that UCN can also be used for nerve grafting. In the advanced neurotization techniques during motor nerve reconstructions, it was proposed that even UCN can be effectively used as a nerve graft. UCN can be used for the reinnervation in denervated biceps brachii due to brachial plexus palsies and it can also be used for the direct nerve transfer (2).

The distal triceps injuries are rare, however they are seen sometimes in middle aged males particularly in weight-lifters. Because of its relatively rare occurrence, the triceps pathologies may lead to difficulty in the initial diagnosis and management. Snapping of triceps is more common than its tendon rupture in younger population and it is often associated with the ulnar dislocation (25). The distal third of the triceps contains independent motor units with an easy access and it is suggested to be ideal for the motor reconstructions with lesser morbidity (2). UCN can be entrapped due to compression, snapping, rubbing and slipping (4). In interpreting the electrophysiological tests and radiological images, UCN is important (4). Good health and well-being are among the sustainable development goals which are adopted by the BRIC countries and India is among them. In this context, the morphological and topographic knowledge of UCN is important to prevent its iatrogenic injury during the medial and posterior approaches of elbow joint surgery. The present study has certain limitations as the specimens used were from different individuals and hence side-based comparison was not feasible. Since this is a cadaveric study it is prone for alterations in the anatomical structures.

\section{CONCLUSIONS}

The present study is suggestive of higher frequency of UCN in Indian ethnic population as it was observed in $83.8 \%$ of our anatomical specimens. This study provides the data about the origin, course and termination of $\mathrm{UCN}$, which are useful to the clinicians and operating surgeons to prevent its iatrogenic injury during the shoulder and elbow surgeries. UCN can be used to re-innervate the muscles in case of brachial plexus injuries and reestablishing the flexion of the elbow. Medial head of triceps brachii is the favorite muscle for the plastic surgery, which is being used for the muscle transfer procedures as this is a bulky muscle with multiple innervation. Nerve transfers are preferred over the nerve grafting for the brachial plexus reconstruction and peripheral nerve injuries as it gives better outcomes. In this context, the detailed knowledge about the morphology and topography of UCN can help the orthopedic surgeons during the procedures like muscle transfer, nerve transfer and nerve grafting. 


\section{ACKNOWLEDGEMENTS}

The authors thank Dr. Soubhagya Ranjan Nayak, Professor of Anatomy, College of Medicine and JNM Hospital, Kalyani, Nadia, West Bengal, India for the help offered during the commencement of this study.

\section{CONFLICT OF INTERESTS}

The authors declare that they have no conflict of interests.

\section{REFERENCES}

1. Miguel-Perez MI, Combalia A, Arandes JM. Abnormal innervation of the triceps brachii muscle by the ulnar nerve. J Hand Surg Eur 2010;35:430-431.

2. Bekler H, Wolfe VM, Rosenwasser MP. A cadaveric study of ulnar nerve innervation of the medial head of the triceps brachii. Clin Orthop Relat Res 2009;647:235-238.

3. Loukas M, Bellary SS, Yuzbasioglu N, et al. Ulnar nerve innervation of the medial head of the triceps brachii muscle: a cadaveric study. Clin Anat. 2013;26:1028-1030.

4. Develi S. Co-innervation of triceps brachii muscle with variant branch of ulnar nerve Turk Neurosurg. 2018;28:949-953.

5. Cho SH, Chung IH, Lee UY. Relationship between the ulnar nerve and the branches of the radial nerve to the medial head of the triceps brachii muscle. Clin Anat 2019; 32:137-142.

6. Jain DA, Kumar ST, Shetty N. Ulnar nerve innervation to triceps: A cadaveric study and a technical note on partial triceps to biceps transfer. Indian J Orthop. 2019;53:353-356.

7. Ramström M. Investigations on the innervation of the medial head of the triceps brachii muscle. Anat Anz 1918;19:420-431

8. Rezzouk J, Durandeau A, Vital JM, Fabre T. Long head of the triceps brachii in axillary nerve injury: anatomical and clinical aspects. Rev Chir Orthop Reparatrice Appar Mot 2002;88: 561-564.

9. Naidu S, Lim A, Poh LK, Kumar VP. Long head of the triceps transfer for elbow flexion. Plast Reconstr Surg 2007;119:45e-7e.

10. Ozer H, Açar HI, Cömert A, et al. Course of the innervation supply of medial head of triceps muscle and anconeus muscle at the posterior aspect of humerus (anatomical study). Arch Orthop Trauma Surg 2006;126:549-553.
11. Chaware PN, Santoshi JA, Patel M, et al. Surgical Implications of Innervation Pattern of the Triceps Muscle: A Cadaveric Study. J Hand Microsurg 2018;10:139-142.

12. Hollinshead HW. The Back and Limbs. In: Anatomy for Surgeons, Vol. 3, 2 $2^{\text {nd }}$ ed. Philadelphia; Harper \& Row 1969;376-378.

13. Johnson D, Ellis H. Upper Arm. In: Standring S, Williams PL, Bannister LH, et al, editors. Gray's Anatomy: The Anatomical Basis of Clinical Practice, 39 ${ }^{\text {th }}$ ed. Edinburgh; Churchill Livingstone 2005;857.

14. Sinnatamby CS. In: Last's Anatomy: Regional and Applied, $11^{\text {th }}$ ed. Edinburgh; Churchill Livingstone 2006;99.

15. Taheri MMH, Afshar M. Connection between radial and ulnar nerves at humeral level and its clinical significance: A cadaveric case report. Int. J. Morphol 2015;33:1559-1562.

16. Pascual-Font A, Vazquez T, Marco F, et al. Ulnar nerve innervation of the triceps muscle: real or apparent? An anatomic study. Clin Orthop Relat Res 2013;471:1887-1893.

17. Linell EA. The distribution of nerves in the upper limb, with reference to variabilities and their clinical significance. J Anat 1921;55:79-112.

18. Cavalheiro CS, Filho MR, Rozas J, et al. Anatomical study on the innervation of the elbow capsule. Rev Bras Ortop 2015;50:673-679.

19. Bertelli JA. Brachialis muscle transfer to the forearm muscles in obstetric brachial plexus palsy. J Hand Surg $\mathrm{Br}$ 2006;31:261-265.

20. Chepla KJ, Bafus BT. Transfer of a radial nerve branch to the brachialis nerve for restoration of elbow flexion. Tech Hand Up Extrem Surg. 2018;22:65-67.

21. Padulo J, Oliva F, Frizziero A, Maffulli N. Muscles, Ligaments and Tendons Journal - Basic principles and recommendations in clinical and field Science Research: 2016 Update. Muscles Ligaments Tendons J 2016;6:1-5.

22. Sunderland S. Metrical and nonmetrical features of the muscular branches of the radial nerve. J Comp Neurol 1946;85:93-111.

23. Madsen M, Marx RG, Millett PJ, et al. Surgical anatomy of the triceps brachii tendon: anatomical study and clinical correlation. Am J Sports Med. 2006;34:1839-1843.

24. Leechavengvongs S, Witoonchart K, Uerpairojkit C, et al. Nerve transfer to deltoid muscle using the nerve to the long head of triceps. Part II: a report of seven cases. J Hand Surg Am 2003;28:633-638.

25. Spinner RJ, Goldner RD. Snapping of the medial head of the triceps: diagnosis and treatment. Tech Hand Up Extrem Surg 2002;6:91-97. 